La Revue

des Droits

de l'Homme

\section{La Revue des droits de l'homme}

Revue du Centre de recherches et d'études sur les droits fondamentaux

$1 \mid 2012$

Revue des droits de l'homme $-\mathrm{N}^{\circ} 1$

\title{
Chapitre 2. Les droits sociaux dans l'ordre juridique de l'Union Européenne
}

Entre instrumentalisation et « fondamentalisation »

\section{Myriam Benlolo-Carabot}

\section{(2) OpenEdition}

Journals

Édition électronique

URL : http://journals.openedition.org/revdh/120

DOI : $10.4000 /$ revdh. 120

ISSN : 2264-119X

Éditeur

Centre de recherches et d'études sur les droits fondamentaux

Édition imprimée

Date de publication : 1 juin 2012

Pagination : 84-102

Référence électronique

Myriam Benlolo-Carabot, « Chapitre 2. Les droits sociaux dans l'ordre juridique de I'Union Européenne ", La Revue des droits de l'homme [En ligne], 1 | 2012, mis en ligne le 30 juin 2012, consulté le 08 juillet 2020. URL : http://journals.openedition.org/revdh/120 ; DOI : https://doi.org/10.4000/revdh.120 
CHAPITRE 2

\author{
LES DROITS SOCIAUX DANS L'ORDRE JURIDIQUE DE \\ L'UNION EUROPÉENNE. ENTRE INSTRUMENTALISATION \\ ET « FONDAMENTALISATION »?
}

\title{
MYRIAM BENLOLO-CARABOT
}

Processus d'intégration économique, la construction communautaire n'a pas au départ placé comme priorité affichée la promotion d'une Europe sociale. Les réticences étatiques à transférer aux Communautés ou à l'Union des compétences significatives dans ce domaine ont rendu sans objet dans l'ordre juridique communautaire les controverses et enjeux connus des ordres juridiques internes, comme la justiciabilité, ou la définition même des droits sociaux. Dans ce contexte, on ne saurait s'étonner de la rareté, jusqu'à une période récente, des études portant sur la prise en compte des droits sociaux dans l'ordre juridique communautaire, la question étant généralement englobée dans une réflexion plus générale, soit dans une perspective internationale ${ }^{271}$, soit dans une approche comparative entre les droits nationaux et l'Union européenne (UE) ${ }^{272}$.

Les évolutions substantielles des traités constitutifs des Communautés et de l'UE, et notamment leur recentrage autour du citoyen européen ${ }^{273}$ et de la protection des droits fondamentaux ${ }^{274}$, ont cependant entraîné une mutation du discours doctrinal, qui vise désormais à rendre compte tant de la consistance que de la spécificité d'une conception communautaire des droits sociaux ${ }^{275}$. Portée

271 Nikitas ALIPRANTIS, Les droits sociaux dans les instruments européens et internationaux. Défis à l'échelle mondiale, Bruylant, 2009; Julia ILIOPOULOS-STRANGAS, Perspectives nationales et internationales des droits sociaux, Bruylant, 2009 ; Jean-François FLAUSS, Droits sociaux et droit européen, Bruxelles, Bruylant 2003.

272 Laurence GAY, Emmanuelle MAZUYER, Dominique NAZET-ALLOUCHE, Les droits sociaux fondamentaux. Entre droits nationaux et droits européen, Bruylant, 2006 ; Julia ILIOPOULOSSTRANGAS, La protection des droits sociaux fondamentaux dans les États membres de l'Union européenne. Étude comparée, Bruylant, 2000.

${ }^{273}$ La citoyenneté européenne est consacrée dans le traité de Maastricht en 1992 (articles 8 à $8 \mathrm{E}$ du traité instituant la Communauté européenne (TCE). Elle occupe aujourd'hui une place centrale dans le traité sur l'UE tel que révisé par le traité de Lisbonne, V. l'article 9 du TUE et la deuxième partie du traité sur le fonctionnement de l'UE (TFUE), "Non-discrimination et citoyenneté de l'Union ", articles 18 à 25.

274 Article 2 du TUE.

275 V. par exemple Catherine PELISSIER, La protection des droits économiques et sociaux fondamentaux dans la Communauté européenne, thèse de doctorat, Université de Montpellier, 2001 ; Sandrine MAILLARD, L'émergence de la citoyenneté sociale européenne, PU Aix-enProvence, 2008, 472 p. ; Claire MARZO, La dimension sociale de la citoyenneté européenne, thèse de doctorat, Institut universitaire européen, 2009, 717 p. 
par la Cour de justice de l'UE, qui tient dans ce processus un rôle tout à fait déterminant, l'affirmation des droits sociaux dans l'ordre juridique de l'UE reste cependant très ambigüe. Instruments d'approfondissement du marché intérieur et des libertés économiques ou fondements d'une Union centrée sur l'Homme, les droits sociaux tels qu'appréhendés par la doctrine et le juge communautaires apparaissent comme un révélateur des tensions caractéristiques de cet ordre juridique singulier qu'est l'UE.

\section{Les droits sociaux, instruments d'approfondissement du marché intérieur dans l’ordre juridique de l'Union européenne}

Les difficultés à établir une compétence de l'Union en matière sociale ont pour conséquence de reléguer les droits sociaux au rang de "parents pauvres" du processus d'intégration : ce n'est que progressivement et avec réticences que sont inscrits dans les traités des compétences et des objectifs sociaux (A). Dans ce contexte juridique, la fonction des droits sociaux ne peut être analysée que de manière indirecte et fonctionnelle, comme un corollaire indispensable à la réalisation du marché intérieur (B).

\section{A. Une prise en compte laborieuse des droits sociaux dans l'ordre juridique de l'UE}

S’il faut prendre acte de l'évolution substantielle des traités constitutifs dans le domaine social (1), la prise en compte des droits sociaux dans l'ordre juridique de l'UE reste empreinte d'une grande ambigüité (2).

1) Une prise en compte progressive des droits sociaux dans le droit primaire de l'UE

Les relations entre le droit communautaire et les droits sociaux ont longtemps été jugées sévèrement par la doctrine, qui n’a eu de cesse de dénoncer une prise en compte très insuffisante des droits sociaux et la part trop belle réservée à l'économie. Le droit primaire originaire est en effet d'un silence assourdissant sur les questions sociales. L'article premier du TCEE, tel qu'il est rédigé en 1957, dispose que «[1]a Communauté a pour mission, par l'établissement d'un marché commun et par le rapprochement progressif des politiques économiques des Etats membres, de promouvoir un développement harmonieux des activités économiques dans l'ensemble de la Communauté, une expansion continue et équilibrée, une stabilité accrue, un relèvement accéléré du niveau de vie et des relations plus étroites entre les Etats qu'elle réunit ". La seule référence au social figure dans le Préambule, qui dispose que les Etats membres sont "décidés à assurer par une action commune le progrès économique et social de leur pays en éliminant les barrières qui divisent l'Europe ", et qui fixe comme but essentiel du traité l'" amélioration constante des conditions de vie et d'emploi de leurs peuples ». 
La signature de l'Acte Unique européen, qui assigne pour la première fois des objectifs sociaux à la Communauté, le protocole social annexé au traité de Maastricht, puis surtout la signature du traité d'Amsterdam, marquent des étapes importantes dans l'élaboration d'une politique sociale communautaire. "Eléments objectifs [qui] obligent à revisiter des problématiques antérieures ${ }^{276}$, les innovations des traités successifs et notamment, la référence récurrente aux droits sociaux fondamentaux opérée par le traité d'Amsterdam, avec un renvoi à la Charte sociale de 1961 et celle de 1989, permettent « une occupation de plus en plus tangible du terrain de la régulation normative par le droit dérivé ${ }^{277}$. Les institutions communautaires sont en effet habilitées à agir par voie de directives dans des domaines importants (conditions de travail pour protéger la santé et la sécurité des travailleurs, information et consultation, intégration des personnes exclues du marché du travail, égalité de traitement entre les hommes et les femmes), construisant ainsi indéniablement un droit social communautaire 278 .

Le traité de Lisbonne étoffe substantiellement les références aux objectifs sociaux. L'article 9 du traité sur le fonctionnement de l'UE (TFUE) dispose que l'Union « dans la définition et la mise en œuvre de ses politiques et actions, [...] prend en compte les exigences liées à la promotion d'un niveau d'emploi élevé, à la garantie d'une protection sociale adéquate, à la lutte contre l'exclusion sociale ainsi qu'à un niveau élevé d'éducation, de formation et de protection de la santé humaine $"$.

\section{2) Une prise en compte hésitante}

Les évolutions du droit primaire ne lèvent pas, loin de là, toutes les ambiguïtés quant à l'effectivité des droits sociaux dans l'ordre juridique de l'Union. En effet, la politique sociale de l'Union ne paraît pas véritablement motivée par un objectif de protection des "droits sociaux fondamentaux ", tant apparaît vague la référence à ces derniers et large la marge de manœuvre laissée aux autorités nationales. Pour de nombreux auteurs, l'article $136 \mathrm{du}$ TCE se contente d'une référence aux droits sociaux fondamentaux sans en

\footnotetext{
276 Jean-François AKANDJI-KOMBE, "Charte sociale et droit communautaire », La Charte sociale européenne, Bruylant, 2001, pp. 149-177.

277 Marie-Ange MOREAU, "L'utilisation des droits sociaux fondamentaux par les travailleurs dans l'Union européenne", in Jean.-Yves CHEROT, Tobias VAN REENEN (dir.), Les droits sociaux fondamentaux à l'âge de la mondialisation, PU Aix Marseille, 2005, pp. 187-188.

278 Parmi les instruments normatifs les plus importants, V. la directive $n^{\circ}$ 76/207/CEE du Conseil du 9 février 1976, relative à l'égalité de traitement entre les hommes et les femmes en ce qui concerne l'accès à l'emploi, à la formation et à la promotion professionnelles, et les conditions de travail, JO L 39 du 14 février 1976, p. 40 ; Directive n 89/391/CEE du Conseil du 12 juin 1989, concernant la mise en œuvre de mesures visant à promouvoir l'amélioration de la sécurité et de la santé des travailleurs au travail, JO L 183 du 29 juin 1989, p. 1 (ainsi que les nombreuses directives particulières prises sur la base de cette directive) ; Directive $n^{\circ}$ 98/59/CE du Conseil du 20 juillet 1998, concernant le rapprochement des législations des Etats membres relatives aux licenciements collectifs, JO L 225 du 12 août 1998, p. 16. Pour une vue d'ensemble, V. Frédéric BARON, Marché intérieur et droit social dans l'Union européenne, PUAM, 1998, 613 p.
} 
imposer le respect ${ }^{279}$. Apparaît ici une problématique très spécifique à l'ordre juridique communautaire, celle de la compétence des institutions communautaires pour prendre des mesures dans le domaine social. Parce que le postulat de départ est que l'on ne peut consacrer dans l'ordre juridique communautaire que des droits qui présentent un lien étroit avec une compétence existante de la Communauté européenne, l'effet direct de ces droits est nié : de ce fait, devraient être écartés les droits pour lesquels l'Union n'a aucune compétence, comme le droit au logement ou le droit au maintien de la sécurité sociale. Or, exiger un lien entre le droit et l'existence d'une compétence communautaire ne saurait être admis que pour la garantie effective du droit et non sa reconnaissance ${ }^{280}$. En ce qui concerne donc le respect des droits, qui concerne tous les droits sociaux, il existerait une obligation générale imposée aux autorités publiques de ne pas méconnaitre certaines exigences minimales.

Quelles que soient l'étendue et la nature actuelles de la compétence communautaire, la logique économique des traités fondateurs continue d'imprégner la prise en considération des droits sociaux. L'article 136 du TCE, qui énonce ambitieusement les objectifs sociaux de la Communauté et des Etats membres, situe leur réalisation dans la perspective du marché commun. Aux termes de l'article 136, alinéa 3, l'évolution vers la réalisation de ces objectifs "résultera tant $\mathrm{du}$ fonctionnement du marché commun, qui favorisera l'harmonisation des systèmes sociaux, que des procédures prévues par le présent traité et du rapprochement des dispositions législatives, réglementaires et administratives ». La réalisation des droits sociaux n'est ainsi envisagée que comme une résultante du marché commun, dont le bon fonctionnement reste l'objectif central dans les traités communautaires. Elément tout à fait important, l'article 136, devenu article 151, reste inchangé dans ses termes dans le TFUE. L'instrumentalisation des droits sociaux dans l'approfondissement du marché unique reste donc tout à fait d'actualité, comme l'atteste le raisonnement du juge de l'Union européenne ${ }^{281}$.

\section{B. Les droits sociaux, corollaires du marché intérieur pour le juge de l'UE}

$\mathrm{Si}$, très tôt, le juge communautaire n'a eu de cesse d'étendre le champ d'application du droit communautaire à certaines prestations sociales (1), il est réticent à admettre leur pleine effectivité en dehors des finalités économiques des traités fondateurs (2).

\footnotetext{
${ }^{279}$ Ricardo GOSALBO BONO, «Les politiques et actions communautaires », RTDE, 1997, p. 780, spéc. p. 784 ; Catherine HAGUENAU-MOIZARD, "Le Traité d'Amsterdam, une négociation inachevée ", RMC, n 417, avril 1998, p. 249. Pour Jean-François AKANDJI-KOMBE, la Charte sociale est promue non pas en tant que mesure de validité du droit dérivé, mais comme base ou point d'appui de la politique sociale communautaire, précit.

280 Jean-François AKANDJI-KOMBE, précit.

${ }^{281}$ Bien que concurrencée par d'autres logiques, visant à «fondamentaliser» les droits sociaux, V. infra, II.
} 
1) La volonté du juge de l'Union d'inclure les droits sociaux dans le champ d'application matériel des traités

Même si le juge communautaire n'a eu de cesse de confirmer le caractère programmatique des objectifs sociaux énoncés dans l'Acte unique européen ${ }^{282}$, il a dans le même temps concilié les objectifs de réalisation du marché intérieur et de protection des droits sociaux. Démontrés par certains auteurs ${ }^{283}$, la compatibilité entre le processus d'intégration et la protection nécessaire de certains droits sociaux, voire même le caractère indispensable de la garantie des droits sociaux pour l'approfondissement du marché commun, ont été illustrés par une jurisprudence fournie de la Cour de justice des Communautés européennes (CJCE). Dès 1964, l'arrêt Unger, qui donne la possibilité à une citoyenne hollandaise sans emploi d'accéder aux services sociaux de la République fédérale d'Allemagne où elle séjourne pour convenances personnelles mais non pour la recherche ou l'occupation d'un emploi ${ }^{284}$, oblige le Conseil des ministres à substituer au Règlement 3 «concernant la sécurité sociale des travailleurs migrants » ${ }^{285}$ le Règlement 1408/71 relatif "aux travailleurs qui se déplacent à l'intérieur de la Communauté ${ }^{286}$. La modification n'est pas seulement sémantique: elle permet de prendre acte de l'importance progressivement donnée par la Cour de Luxembourg au simple déplacement dans l'espace communautaire, quels que soient les motifs à l'origine de celuici $^{287}$, y compris donc s'il s'agit de rechercher une protection sociale adéquate.

Les domaines dans lesquels un tel mouvement est perceptible sont très nombreux: donnant une interprétation particulièrement constructive $\mathrm{du}$ règlement 1612/68 relatif à la libre circulation des travailleurs ${ }^{288}$, qui dispose

282 Pour un exemple particulièrement significatif, V. CJCE, 29 septembre 1987, Giménez Zaera / Instituto Nacional de la Seguridad Social et Tesorería General de la Seguridad Social, aff. 126/86, Rec. 1987, p. 3697.

283 V., outre les ouvrages de droit matériel communautaire : Frédéric BARON, Marché intérieur et droit social dans l'UE, PUAM, 1998 ; Lammy BETTEN, Delma MACDEVITT, The Protection of Fundamental Social Rights in the EU, Boston, Kluwer Law international, 1996 ; Jean-Pierre PUISSOCHET, "La progression des droits sociaux dans une Communauté économique, les apports récents de la CJCE», in Mélanges en l'honneur de Jean Pélissier, Dalloz, p. 433; Wolfgang DÄUBLER, "Les droits sociaux dans l'UE. De la symbolique à la pratique politique ?", in Mario TELÒ, Démocratie et construction européenne, Editions de l'ULB, 1995, p. 314 ; MarieAnge MOREAU, "L'utilisation des droits sociaux fondamentaux par les travailleurs dans l'UE », in Jean-Yves CHEROT, Tobias VAN REENEN, Les droits sociaux fondamentaux à l'âge de la mondialisation, PUAM, 2005, p. 175 ; Sophie ROBIN-OLIVIER, « La référence aux droits sociaux fondamentaux dans le traité d’Amsterdam », Droit social, 1999, pp. 609 et s.

${ }^{284}$ CJCE, 19 mars 1964, Unger, aff. 75/63, Rec., p. 349.

285 Règlement (CEE) du Conseil $n^{\circ} 3$ du 25 septembre 1958, concernant la sécurité sociale des travailleurs migrants, JOCE B 030, 16 décembre 1958, p. 561, modifié par le Règlement (CEE) du Conseil n²4/64 du 10 mars 1964, JOCE n 47, 18 mars 1964, p. 746.

286 Règlement (CEE) n 1408/71 du Conseil, du 14 juin 1971, relatif à l'application des régimes de sécurité sociale aux travailleurs salariés, aux travailleurs non salariés et aux membres de leur famille qui se déplacent à l'intérieur de la Communauté, JOCE L 149, 5 juillet 1971, p. 2.

287 Sur ces jurisprudences, V. Patrick DOLLAT, Libre circulation des personnes et citoyenneté européenne, Bruylant, 1998, p. 102 ; Myriam BENLOLO CARABOT, Les fondements juridiques de la citoyenneté européenne, Bruylant, 2006, p. 485.

288 Règlement $\mathrm{n}^{\circ} 1612 / 68$ du Conseil, du 15 octobre 1968, relatif à la libre circulation des travailleurs à l'intérieur de la Communauté, JO L 257 du 19 octobre 1968, p. 2. 
notamment dans son préambule que " la mobilité de la main d'œuvre dans la Communauté doit être pour le travailleur un des moyens qui lui garantissent la possibilité d'améliorer ses conditions de vie et de travail et de faciliter sa promotion sociale ", la Cour a par exemple estimé que les membres de la famille du ressortissant communautaire avaient droit à un enseignement général, un apprentissage et une formation professionnelle dans les mêmes conditions que les ressortissants de l'État membre d'accueil à la condition qu'ils résident sur le territoire ${ }^{289}$. Les membres de la famille $\mathrm{e}^{290}$ ont aussi accès aux avantages sociaux accordés au ressortissant communautaire ${ }^{291}$, ces " avantages sociaux " étant eux-mêmes interprétés particulièrement souplement292. Seront considérés comme des avantages sociaux par le juge communautaire des aides aussi différentes qu'une allocation de maternité293, une allocation pour handicapés adultes ${ }^{294}$, des allocations d'attente ${ }^{295}$, ou encore un revenu garanti aux personnes âgées ${ }^{296}$. L'extension très singulière du principe d'égalité de traitement porte ce mouvement d'inclusion des droits sociaux dans l'ordre juridique communautaire, et ce, dès les premières années du processus d'intégration.

Ce mouvement très remarquable n'entraîne pourtant pas une réflexion élaborée du juge communautaire sur la «fondamentalité" de certains droits sociaux. Perçus comme des corollaires nécessaires du processus d'intégration, les droits sociaux ont peine à s'imposer comme tels dans le raisonnement du juge, souvent prompt à favoriser les libertés économiques de libre circulation, promues au rang de libertés fondamentales. Il y a là un décalage tout à fait net avec le discours doctrinal, celui-ci s'efforçant, tant bien que mal ${ }^{297}$, de systématiser l'existence et la consistance juridique de droits sociaux fondamentaux dans l'ordre juridique communautaire.

289 V. entre autres, sur la question des bourses et allocations, CJCE, 3 juillet 1974, Casagrande / Landeshauptstadt München, aff. 9/74, Rec. 1974, p. 773.

290 Sur les membres de la famille, V. entre autres Constança DIAS URBANO DE SOUSA, " Le droit des membres de la famille du citoyen de l'Union européenne de circuler et de séjourner sur le territoire des États membres, dans la directive 2004/38/CE ", in Jean-Yves CARLIER, Elspeth GUILD (dir.), L'avenir de la libre circulation des personnes dans l’U.E., Bruylant, 2006, pp. 103125.

291 CJCE, 30 septembre 1975, Cristini / S.N.C.F., aff. 32/75, Rec. 1975, p. 1085.

292 Pour une analyse d'ensemble, v., entre autres, Jean-Pierre PUISSOCHET, « La progression des droits sociaux dans une Communauté économique, les apports récents de la CJCE ", in Mélanges en l'honneur de Jean Pélissier, Dalloz, p. 433; Claire MARZO, La dimension sociale de la citoyenneté européenne, précit., pp. 285-287.

293 CJCE, 10 mars 1993, Commission / Luxembourg, aff. C-111/91, Rec. 1993, p. I-817.

${ }^{294}$ CJCE, 27 mai 1993, Schmid / État belge, aff. C-310/91, Rec. 1993, p. I-3011.

${ }^{295}$ CJCE, 20 juin 1985, ONEM / Deak, aff. 94/84, Rec. 1985, p. 1873.

296 CJCE, 12 juillet 1984, Castelli / ONTPS, aff. 261/83, Rec. 1984, p. 3199.

297 V., pour une analyse du discours doctrinal communautariste, Myriam BENLOLO-CARABOT, Béatrice DELZANGLES, Selma JOSSO, « Les droits sociaux appréhendés par la doctrine de droit communautaire. Renouvellement ou fin d'une catégorie juridique ?», consultable en ligne: http://www.droits-sociaux.u-paris10.fr/index.php?id=143 
2) Les réticences du juge de l'Union à assurer l'effectivité des "droits sociaux fondamentaux "

Les réticences du juge de l'Union à donner une effectivité réelle aux droits sociaux sont perceptibles tant dans le traitement réservé à certains instruments juridiques communautaires ou internationaux, que dans la conciliation que le juge opère entre les libertés dans la réalisation du marché commun. Quant au premier point, il faut noter la relative rareté des prises de position jurisprudentielles faisant référence à la Charte sociale européenne et à la Charte communautaire des droits sociaux fondamentaux. Dénoncée par la doctrine $^{298}$, cette indifférence confirme les hésitations du juge à consacrer une catégorie juridiquement bien établie de "droits sociaux fondamentaux". Dans l'arrêt Blaizot, prononcé le 2 février 1988 au sujet de l'interprétation de la notion de "formation professionnelle ", la Cour considère l'enseignement universitaire comme un moyen de réaliser les objectifs poursuivis par la Communauté en matière d'éducation, citant la Charte sociale européenne en appui à son raisonnement ${ }^{299}$. Plus récemment, la Cour a accepté de considérer en examinant une directive communautaire que "le législateur communautaire a entendu se conformer aux objectifs de la charte communautaire des droits sociaux fondamentaux des travailleurs. (...)». La Cour ajoute que "L'article 136 se réfère également à la Charte sociale européenne (...) ${ }^{300}$. D’une manière plus explicite, la Cour a fait référence à la Charte communautaire des droits sociaux fondamentaux au sujet du congé parental, reconnaissant «le caractère fondamental de ce droit social $[\ldots] »^{301}$.

Quant au deuxième point, la doctrine a souvent et à juste titre mis l'accent sur la confrontation entre la logique économique de marché et les droits sociaux $^{302}$. Les libertés fondamentales sont souvent analysées comme des dangers pour les législations nationales protectrices des droits sociaux. En témoignent les très nombreux arrêts de la CJCE, dans lesquels la juridiction communautaire a pu privilégier la réalisation d'une liberté économique au détriment d'un avantage social ${ }^{303}$. La problématique reste tout à fait actuelle,

298 Eliane VOGEL POLSKY, "L'Europe sociale de l'an 2000, la Charte sociale et le système communautaire ", Charte sociale européenne, Actes du Colloque de Grenade, 26-27 octobre 1987, Conseil de l'Europe, 1989, pp. 46 et s.

299 CJCE, 2 février 1988, Blaizot c. Université de Liège e.a., aff. 24/86, Rec., p. 379, point 17.

300 CJCE, 20 septembre 2007, Sari Kiiski, aff. C-116/06, Rec., point 48. V. aussi Tribunal de la fonction publique, 26 octobre 2006, Pia Landgren, aff. F-1/05, Rec., 2006, p. I-A-1-123, points 69 à 72 .

301 CJUE, 16 septembre 2010, Zoi Chatzi, aff. C-149/10, point 63. V. aussi l'arrêt du 10 juin 2010 de la CJUE, INPS c. Bruno et Pettini, aff. C-395/08 et C-396/08, points 31 et 32 : la Cour rappelle que l'article 136 renvoie à la Charte sociale européenne, et estime qu'eu égard " à ces objectifs, la clause [...] doit être comprise comme exprimant un principe de droit social de l'Union qui ne saurait être interprété de manière restrictive ".

302 Alain BUZELAY (dir.), Une Europe sociale: pourquoi... jusqu'où ?, Nomos Verlagsgesellschaft, 1995, notamment la contribution de Gérard LYON-CAEN, "Le droit social dans la CE », pp. 83125.

${ }^{303}$ Quelques exemples parmi tant d'autres : question des horaires de travail des employés (CJCE, aff. 145/88, Sunday Training) ou problème du statut monopolistique du travail sur les docks incompatible avec l'art. 30 du traité (CJCE, aff. C-179/90, Merci Convenzionali Porto di Genova). 
même si certains conflits semblent moins vivaces ${ }^{304}$, comme l'attestent les prises de position récentes après les arrêts Laval et Viking, prononcés par la CJCE en décembre 2007 ${ }^{305}$. Dans sa tentative de conciliation de l'exercice des droits syndicaux et de la libre prestation de services, la Cour reconnaît que le droit de grève est un droit fondamental. Largement approuvé sur ce point, le raisonnement de la juridiction communautaire se trouve cependant sévèrement critiqué par la doctrine quant aux limitations posées dans l'exercice de ce droit afin de ne pas entraver le libre établissement ou la libre prestation de services d'une entreprise ${ }^{306}$. Critiquée pour son dirigisme, la Cour peut l'être aussi paradoxalement quand elle renvoie au juge national le soin de faire certains arbitrages, masquant mal son malaise et ses difficultés à concilier certaines exigences sociales et l'impact de la libéralisation du marché européen, dans des domaines sensibles comme l'éducation ${ }^{307}$ ou la santé ${ }^{308}$.

Dans ce contexte juridique, il est bien difficile pour la doctrine de systématiser l"existence dans l'ordre juridique de l'UE d'un corpus indiscutable de droits sociaux fondamentaux. Coexistent plutôt de multiples constructions doctrinales, proposant des catalogues de droits considérés comme des droits sociaux fondamentaux (égalité entre les hommes et les femmes, libre circulation des travailleurs, droit à la mobilité professionnelle, droit d'entrée et de séjour) ${ }^{309}$. Pour certains auteurs, il faut entendre par "droits sociaux fondamentaux" certains "droits sociaux minimaux" formant un plancher minimum commun ${ }^{310}$. Pour d'autres, comme le Professeur Rodière, les droits qualifiés de fondamentaux sont ceux respectés par et dans l'ordre juridique communautaire. Cette donnée générale «s'étend aux droit sociaux fondamentaux ${ }^{311}$, mais sans que l'on puisse établir de catalogue. Les oppositions doctrinales se cristallisent bien sûr sur l'étendue et le contenu de ce catalogue, notamment sur l'inclusion des " droits du travailleur " dans les droits sociaux. De manière tout à fait frappante, la doctrine ne s'appuie pas sur la Charte communautaire des droits sociaux fondamentaux pour définir le droit

Pour un panorama très complet, V. Miguel POIARES MADURO, «L'équilibre insaisissable entre la liberté économique et les droits sociaux dans l'UE », in Philip ALSTON, L'Union européenne et les droits de l'Homme, Brucelles, Bruylant, 2001, pp. 465-489.

${ }^{304}$ Etienne PATAUT, Sophie ROBIN-OLIVIER, " Europe sociale ou Europe économique ", Revue du droit du travail, février 2008; Alberto ALEMANNO, "A la recherche du juste équilibre entre libertés fondamentales et droits fondamentaux dans le cadre du marché intérieur ", RDUE, 2004, $\mathrm{n}^{\circ}$ 4, p. 709 ; Théodore GEORGOPOULOS, "Libertés fondamentales communautaires et droits fondamentaux européens : le conflit n'aura pas lieu ", LPA, 2004, n 6, p. 11.

305 CJCE, 11 décembre 2007, Vicking, aff. C-438/05; Par ex : Jean-Sylvestre BERGE, Sophie ROBIN-OLIVIER, Introduction au droit européen, PUF, 2008, p. 312.

306 Pour un exemple plus récent, V.. CJUE, 15 juillet 2010, Commission c. Allemagne, aff. C$271 / 08$.

307 V. par ex. CJUE, arrêt du 13 avril 2010, Bressol et Chaverot, aff. 73/08.

308 V. par ex. CJCE, 16 mai 2006, Yvonne Watts, aff. C-372/04, Rec., p. I-4325.

309 Jean-François AKANDJI-KOMBE, «Conseil de l'Europe et droit communautaire », in JeanFrançois AKANDJI-KOMBE, précit., pp. 149-177 ; du même auteur, " Le développement des droits fondamentaux dans les traités ", in Stéphane LECLERC, Jean-François AKANDJI-KOMBE, L'UE et les droits fondamentaux, Bruylant, 1999, pp. 31 et s.

310 Emmanuelle MAZUYER, précit.

311 Pierre RODIERE, Droit social de l'UE, Paris, LGDJ, 2008, p. 139. 
social fondamental, ce texte étant la plupart du temps analysé fort succinctement, parfois de manière ouvertement critique ${ }^{312}$. Déclaration politique dénuée de réelle portée, la Charte de 1989 n'a pour la doctrine qu'un rôle limité ${ }^{313}$.

Des évolutions substantielles du droit de l'Union permettent cependant d'envisager une nouvelle dynamique. La question de la "fondamentalisation" des droits sociaux se trouve désormais posée dans l'ordre juridique de l'Union.

\section{Une "fondamentalisation " ambigüe des droits sociaux dans l'ordre juridique de l'Union européenne}

En fondant désormais l'Union sur le respect des droits de l'Homme, les traités permettent une protection juridictionnelle plus effective de certains droits sociaux (A). L'instauration de la citoyenneté européenne pousse elle aussi, d'une autre manière, à la « fondamentalisation » de ces droits (B).

\section{A. Les droits de l'Homme, fondement nouveau pour une prise en considération des droits sociaux dans l'ordre juridique de l'UE}

Le principe d'égalité entre les hommes et les femmes, et plus encore, la clause générale de non-discrimination, permettent au juge communautaire, et ce, avant que ne soit conférée une valeur juridique contraignante à la Charte des droits fondamentaux, de donner pleine effectivité à certains droits fondamentaux dont la dimension sociale est avérée (1). Quant à la Charte des droits fondamentaux, ses conséquences sur la justiciabilité des droits sociaux paraissent aujourd'hui incertaines, tout en étant riches de potentialités (2).

\section{1) Le principe de non-discrimination, instrument efficace de promotion} des droits sociaux dans l'ordre juridique de l'Union

Retentissant, l'arrêt Defrenne du 8 avril $1976^{314}$ reconnaît l'effet direct de l'article 119 relatif à l'égalité de rémunération entre les hommes et les femmes, amenant la doctrine à saluer la consécration d'une conception globale de la

\footnotetext{
312 Pierre RODIERE note "l'usage - ou l'abus - largement fait de qualificatifs vagues (« juste», " équitable", "suffisant", " décent») ou de principes cadre" dans la charte, précit., p. 151. V. aussi Christopher LINGLE, "The EC Social Charter, social democracy and post-1992 Europe", West European Politics, janvier 1991, pp. 129-138 ; Sophie ROBIN-OLIVIER, "La référence aux droits sociaux fondamentaux dans le traité d'Amsterdam ", Droit social, 1999, pp. 609-621; Jean DUREN, "La Charte communautaire des droits sociaux fondamentaux devant le Parlement", RMCUE, janvier 1991, pp. 19-25 ; Philippa WATSON, "The Community Social Charter ", CMLR, 1991, pp. 37-68 ; Bob HEPPLE, « The Implementation of the Community Charter of fundamental social rights", Modern Law Review, septembre 1990, pp. 643-654.V. cependant pour une approche différente Paul TEAGUE, John GRAHL, "The European Community Social Charter and labor market regulation ", Journal of Public Policy, juin 1991, pp. 207-232.

313 Philippe POCHET, "L'actualité du droit du travail communautaire : la mise en ouvre de la Charte de 1989 », Droit social, 1993, pp. 695-701.

314 CJCE, 8 avril 1976, Defrenne c. Sabena, aff. 43/75, Rec., p. 455.
} 
société, dans laquelle l'économie et le social doivent aller de concert. Pour la Cour de justice en effet, l'article 119 remplit une double finalité, éviter d'une part que des entreprises ayant éliminé les discriminations salariales ne subissent un désavantage concurrentiel par rapport à celles qui n'ont pas encore aboli les discriminations, et assurer d'autre part la réalisation des "objectifs sociaux" de la Communauté, "celle-ci ne se limitant pas à une union économique, mais devant assurer en même temps, par une action commune, le progrès social et poursuivre l'amélioration constante des conditions de vie et d'emploi des peuples européens $»^{315}$. Le principe d'égalité de rémunération fait donc partie "des fondements de la Communauté " ${ }^{316}$. Confirmée par une jurisprudence constante ${ }^{317}$, cette conception permet "d'entendre la dénomination économique comme couvrant une Communauté économique et sociale $»^{318}$.

L’introduction d'une clause générale de non-discrimination dans la version révisée du TCE à Amsterdam (article 13) a permis d'accentuer ce mouvement et de donner compétence à la Communauté, le Conseil statuant à l'unanimité, pour lutter contre toute discrimination fondée sur le sexe, la race ou l'origine ethnique, la religion ou les croyances, un handicap, l'âge ou l'orientation sexuelle. La Cour a pu ainsi développer une jurisprudence audacieuse visant à donner plein effet aux directives adoptées sur ce fondement, notamment la directive $n^{\circ} 2000 / 78$ du 27 novembre 2000 portant création d'un cadre général en faveur de l'égalité de traitement en matière d'emploi et de travail ${ }^{319}$. Dans l'arrêt Mangold, la Cour estime que la directive ne consacre pas elle-même le principe d'égalité de traitement, celui-ci trouvant sa source "dans divers instruments internationaux et les traditions constitutionnelles communes aux Etats membres». Le principe de non-discrimination en fonction de l'âge "doit ainsi être considéré comme un principe général du droit communautaire ${ }^{320}$.

\section{2) Les conséquences de l'adoption de la Charte des droits fondamentaux}

Le processus d'élaboration de la Charte et sa proclamation en 2000 provoquent un renouvellement important du discours doctrinal relatif aux droits sociaux, désormais étudiés comme une catégorie juridique à part entière (a). Les

\footnotetext{
315 Ibid., point 10.

316 Ibid., point 12.

317 Pour un exemple récent, V. CJCE, 6 décembre 2007, Ursula Voß c. Land de Berlin, aff. C300/06, Rec., p. I-10575.

318 Paul REUTER, "Commentaire de l'article 1 ", in Commentaire article par article du TCEE, sous la direction de Vlad CONSTANTINESCO et al., p. 27, note 31.

319 JO L 303, p. 16.

320 CJCE, 22 novembre 2005, Mangold, aff. C-144/04, Rec., p. I-9981, points 75 et 76 . Pour une confirmation, V. CJUE, 19 janvier 2010, Seda Kücükdeveci, aff. C-555/07, arrêt qui fait référence à la Charte des droits fondamentaux (v. infra) et qui pose un raisonnement tout à fait novateur quant à l'effet direct horizontal des directives et ses conséquences sur le rôle du juge national ; V. aussi CJUE, 12 octobre 2010, Andersen, aff. C-499/08; 12 octobre 2010, Rosenbladt, aff. C-49/09.
} 
effets de la Charte sur le raisonnement du juge sont eux plus aléatoires, la pratique jurisprudentielle restant très lacunaire (b).

\section{a) Les conséquences sur le discours doctrinal : une mutation avérée}

La doctrine de droit communautaire, qui pourtant analyse la place et la fonction des droits sociaux dans l'ordre juridique communautaire ${ }^{321}$ s'interroge peu sur la qualification, la définition ou le contenu des droits sociaux jusqu'en 2000, date d'élaboration et de proclamation de la Charte des droits fondamentaux ${ }^{322}$. Ce n'est qu'à partir du moment où le droit social n'est plus conçu comme simple instrument du marché commun, mais aussi comme un droit de l'Homme devant être garanti comme tel, que la doctrine s'interroge sur des problématiques connues des droits internes, comme par exemple le caractère " mixte " de certains droits ${ }^{323}$, à la fois droits civils et politiques et droits sociaux (exemple du droit à la santé, souvent mis en évidence ${ }^{324}$ ), ou l'inclusion de certains droits, comme la protection de l'environnement et celle des consommateurs, dans la catégorie " droits sociaux".

«La question des droits sociaux représente (...) l'enjeu principal de la Charte » ${ }^{325}$. Ces propos, tenus par Guy Braibant, sont largement admis par le reste de la doctrine. Les auteurs relèvent les nombreuses avancées de la Charte dans la manière de traiter les droits sociaux qui ne sont plus relégués au second plan. La distinction classique entre droits civils et politiques et droits sociaux est reprise par la doctrine qui analyse la Charte des droits fondamentaux, du moins comme point de départ du raisonnement et de la démonstration des auteurs ${ }^{326}$. Sont rappelées la thèse générationnelle des droits fondamentaux et les différences de statut et de régime qui séparent les droits civils et politiques

\section{Supra, I.}

322 Longtemps sans valeur juridique contraignante, la Charte fait désormais partie du droit primaire, l'article 6, § 1, du TUE disposant que «l'Union reconnaît les droits, les libertés et les principes énoncés dans la Charte des droits fondamentaux de l'UE [...], laquelle a la même valeur juridique que les traités" ".

${ }^{323}$ Le rapport du Comité des sages de 1996, Pour une Europe des droits civiques et sociaux, constitue une exception notable au désintérêt de la doctrine, avant 2000, pour la distinction traditionnelle entre droits civils et politiques et droits sociaux. Il fait la distinction entre droits pouvant être invoqués immédiatement et droits ayant un caractère programmatoire. Le Comité ne prend cependant pas position sur la question de la justiciabilité des dispositions programmatoires. ${ }^{324}$ Marie-Claire PONTHOREAU, " Le principe de l'indivisibilité des droits : l'apport de la Charte des droits fondamentaux de l'Union européenne à la théorie générale des droits fondamentaux ", RFDA, 2003, p. 930; Jacqueline DUTHEIL DE LA ROCHÈRE, " La Charte des droits fondamentaux de l'Union européenne : quel apport à la protection des droits ? ", Mélanges en l'honneur de Benoît Jeanneau, Les mutations contemporaines du droit public. Paris, Dalloz, 2002, p. 100 ; Philippe GUILLOT, " Le modèle européen de société ", ", in La France face à la Charte des droits fondamentaux de l'Union européenne, Laurence BURGORGUE-LARSEN (dir.), Bruylant 2005, p. 387.

325 Guy BRAIBANT, "Conclusions des actes du Colloque de Strasbourg relatif à la Charte ", in Florence BENOIT-ROHMER, La charte des droits fondamentaux de l'UE, RUDH, 2000, n 1/2, p. 67.

326 Par ex. Guy BRAIBANT, "La Charte des droits fondamentaux », Droit social, 2001, n 1, pp. 69-75 ; Olivier DE SCHUTTER « La contribution de la Charte des droits fondamentaux de l'Union européenne à la garantie des droits sociaux dans l'ordre juridique communautaire", Revue universelle des droits de l'Homme, 2000, fasc. 12, pp. 33-34. 
(DCP) des droits économiques, sociaux et culturels (DESC). Pour autant, les auteurs ne renvoient pas tous aux même références historiques, juridiques ni aux mêmes présupposés. Pour certains, c'est une approche chronologique qui est privilégiée, les auteurs proposant des lectures parfois différentes des catégories et du caractère nouveau des droits consacrés par la Charte ${ }^{327}$. Parfois, la distinction entre DCP et DESC est fondée à la fois sur le droit national et sur le droit international ${ }^{328}$, parfois sur le seul droit international ${ }^{329}$. Tantôt le droit international est accusé de générer une hiérarchie des droits ${ }^{330}$; tantôt, il est valorisé $^{331}$. Les auteurs insistent souvent sur la situation au sein du Conseil de l'Europe, qui a d'abord reconnu les droits civils et politiques (CEDH) puis les droits sociaux (CSE) ${ }^{332}$. D'autres auteurs enfin utilisent les notions de "libertés formelles » et « libertés réelles » ${ }^{333}$ pour renvoyer à la distinction binaire ${ }^{334}$.

Quelles que soient les justifications et explications, la doctrine est en revanche quasi unanime sur la rupture, ou du moins sur l'apparence de rupture, que propose la Charte des droits fondamentaux à propos de la dichotomie entre les droits civils et politiques et les droits sociaux. La Charte énonce en effet le principe d'indivisibilité dans son Préambule: Consciente de son patrimoine spirituel et moral, l'Union se fonde sur les valeurs indivisibles et universelles de dignité humaine, de liberté, d'égalité et de solidarité. Elle est structurée autour de six valeurs, Dignité, Liberté, Egalité, Solidarité, Citoyenneté, Justice. Ces valeurs constituent les liens unissant les droits civils et politiques et les droits sociaux et affirmant leur indivisibilité ${ }^{335}$. Le plan thématique retenu dans la Charte marque ainsi une volonté « de briser avec une tradition divisant, mais aussi hiérarchisant les droits fondamentaux »336. Bien plus, il " met en lumière l'excessif simplisme des catégories traditionnelles»337. La Charte prévoit en outre la possibilité pour l'autorité compétente d'intervenir afin de définir les

\footnotetext{
327 Marie-Claire PONTHOREAU, « Le principe de l'indivisibilité des droits : l'apport de la Charte des droits fondamentaux de l'Union européenne à la théorie générale des droits fondamentaux ", précit., p. 928 ; Guy BRAIBANT, « La Charte des droits fondamentaux », précit., p. 73 ;

328 Pierre RODIERE, "Les droits sociaux fondamentaux face à la Constitution européenne", in Laurence GAY, Emmanuelle MAZUYER, Dominique NAZET-ALLOUCHE, précit., p. 237 ; JeanMarie SERVAIS, Droit social de l’UE, Bruylant, 2008.

329 Guy BRAIBANT, « La Charte des droits fondamentaux », précit., p. 72.

330 Idem.

331 Jean-Marie SERVAIS, précit., p. 54.

332 Par ex. Pierre RODIERE, Droit social de l'Union européenne, précit., p. 141 ; JeanMarie SERVAIS, précit., p. 55.

${ }_{333}$ Distinction marxiste selon laquelle les libertés formelles sont celles qui ne prévoient pas de moyens économiques pour être mises en œuvre et qui, partant, n'ont de contenu que pour la bourgeoisie qui dispose de tels moyens et les libertés réelles qui sont, quant à elles, effectives et qui sont à tous les citoyens.

${ }^{334}$ V. par ex. Jean-Marie SERVAIS, précit., p. 53 ; Marie-Claire PONTHOREAU, « Le principe de l'indivisibilité des droits : l'apport de la Charte des droits fondamentaux de l'Union européenne à la théorie générale des droits fondamentaux ", précit., p. 929.

335 Marie-Claire PONTHOREAU, Ibid., p. 929.

336 Pierre RODIERE, "Les droits sociaux fondamentaux face à la Constitution européenne", précit., p. 237.

337 Marie-Claire PONTHOREAU, « Le principe de l'indivisibilité des droits : l'apport de la Charte des droits fondamentaux de l'Union européenne à la théorie générale des droits fondamentaux ", précit., p. 928.
} 
conditions d'exercice des droits invoqués dans le cadre de l'emploi (liberté professionnelle; liberté d'association syndicale, droit à la protection contre le licenciement injustifié ou abusif, droits des travailleurs migrants à l'égalité de traitement) ou d'apporter à ces droits certaines restrictions ${ }^{338}$. Parce que ces restrictions doivent viser un but légitime et demeurer proportionnées, on a pu estimer que "rien dans ce régime (...) ne distingue fondamentalement ces droits "sociaux" des droits "civils et politiques" »339.

Les conséquences de la Charte sur la justiciabilité des droits sociaux font cependant l'objet de controverses doctrinales importantes, non encore tranchées par le juge de l'Union.

\section{b) Les conséquences de la Charte sur la justiciabilité des droits sociaux: une mutation potentielle?}

La question de la justiciabilité des droits économiques et sociaux " fut au cœur des controverses au moment de l'élaboration de la Charte ${ }^{340}$. La Charte des droits fondamentaux déclare, à la fin de son Préambule, que "l'Union reconnaît les droits, les libertés et les principes énoncés ci-après". Si la distinction entre droits et libertés n'est pas discutée par la doctrine, une grande partie des commentaires relatifs à la Charte porte sur la distinction entre droits et principes qui constitue, pour certains, "l'un des apports les plus originaux " de ce texte ${ }^{341}$. S'il paraît clair en effet pour la doctrine que tous les droits sociaux ne peuvent prétendre à une garantie équivalente ${ }^{342}$, la question de savoir si la distinction entre les droits et les principes recoupe celle entre les droits civils et politiques et les droits sociaux est beaucoup plus délicate. Fruit d'un compromis résultant de la controverse à l'égard des droits sociaux dont la formulation a donné lieu à d'âpres discussions au moment de l'élaboration du texte ${ }^{343}$, la distinction entre droits et principes est soit minimisée ${ }^{344}$, soit utilisée pour

338 Explications relatives à la Charte élaborées sous l'empire de la Première Convention et retouchées sous la direction de la seconde.

339 Olivier DE SCHUTTER, « La contribution de la Charte des droits fondamentaux de l'Union européenne à la garantie des droits sociaux dans l'ordre juridique communautaire ", RUDH, 2000, vol. $12, \mathrm{n}^{\circ} 1 / 2$, p. 42.

340 Laurence BURGORGUE-LARSEN, " Ombres et Lumières de la constitutionnalisation de la Charte des droits fondamentaux de l'Union européenne ", Cahiers de droit européen, 2004, pp. 685 et $\mathrm{s}$.

341 Guy BRAIBANT, " Conclusions des actes du Colloque de Strasbourg relatif à la Charte ", précit., p. 67.

342 Guy BRAIBANT, ibid. : "On ne dit pas "les" droits économiques et sociaux mais 'des' droits économiques et sociaux. Des membres de la Convention qui ne sont pas favorables aux droits sociaux tirent partie de cette rédaction qui n'est certainement pas due au hasard. Cela signifie qu'il faut opérer une sélection ". V. aussi Olivier DE SCHUTTER, qui distingue les "véritables droits créances" des autres droits, "La contribution de la Charte des droits fondamentaux de l'Union européenne à la garantie des droits sociaux dans l'ordre juridique communautaire ", précit., p. 35.

${ }^{343}$ Guy BRAIBANT, ibid., p. 72.

344 «La Charte, en s'articulant autour de six valeurs fondamentales, formalise (...) la déconnexion entre la classification générationnelle et la protection juridique ", c'est-à-dire entre l'idée que les droits civils et politiques, apparus en premiers, obligeraient l'Etat à s'abstenir tandis que les droits sociaux, reconnus après, entraîneraient une obligation d'action, Marie-Claire 
justifier le fait que les droits sociaux «appellent à [des] dispositions complémentaires" au niveau communautaire ou au niveau national ${ }^{345}$, la distinction "réintrodui[sant] (...) un doute sur l'équivalence des droits fondamentaux $»^{346}$ et remettant donc en cause l'indivisibilité des droits affichée par la Charte. Principes "boucliers" contre droits "épées" ${ }^{347}$, justiciabilité « objective " ou " normative ", justiciabilité « indirecte " permettant de distinguer "l’invocabilité réduite" de la "pleine invocabilité »348, "justiciabilité active" contre justiciabilité "médiate» des principes sociaux ${ }^{349}$, renvoyant à une invocabilité par voie d'exception ${ }^{350}$, les termes se multiplient pour rendre compte d'un même malaise, la doctrine peinant et à donner un sens à la distinction, et à mesurer de ce fait l'apport de la Charte ${ }^{351}$. De fait, il n'existe pas de liste de droits ou de principes dans la Charte. Le mot "principe" n'apparaît pas dans la Charte en dehors du Préambule. La Charte emploie le plus souvent le terme de droit, parfois de liberté. Il faut alors se référer aux explications relatives à la Charte pour voir apparaître la notion de " principe».

Devant ces incertitudes, le travail du juge paraît essentiel. Le juge pourrait en effet être en mesure de lutter contre la passivité des institutions communautaires et/ou nationales qui se refuseraient à prendre des mesures de mise en œuvre ${ }^{352}$. L'invocabilité indirecte permettrait ainsi de ne pas faire régresser le niveau de protection sociale déjà atteint dans le cadre communautaire et/ou national, "telle une clause de stand still" (la violation d'un principe déjà " promu " par un acte de droit dérivé pourrait être censurée au regard des exigences de ce principe) ${ }^{353}$. Le principe d’indivisibilité pourrait

PONTHOREAU, " Le principe de l'indivisibilité des droits : l'apport de la Charte des droits fondamentaux de l'Union européenne à la théorie générale des droits fondamentaux », précit., p. 930.

345 Jacqueline DUTHEIL DE LA ROCHÈRE, " La place de la Charte des droits fondamentaux de l'Union dans la Constitution européenne », précit., p. 238.

346 Pierre RODIERE, "Les droits sociaux fondamentaux face à la Constitution européenne ", précit., p. 237.

${ }^{347}$ Laurence BURGORGUE-LARSEN, « Ombres et Lumières... ", précit., p. 689.

348 Pierre RODIERE, "Les droits sociaux fondamentaux face à la Constitution européenne", précit., p. 249.

${ }^{349}$ Laurence BURGORGUE-LARSEN, « Ombres et Lumières... », précit., p. 689.

350 Elle permet d'attaquer une norme, une loi, une directive ou encore un règlement européen devant le juge administratif saisi par voie d'exception et de soutenir que cette norme viole un des principes affirmés dans la Charte.

${ }^{351}$ La différence de justiciabilité entre les droits et les principes peut aussi être remise en cause pour elle-même: pour Olivier DE SCHUTTER, les principes sociaux sont plus que de simples " objectifs purement programmatiques" et il convient "de présumer " leur justiciabilité ", "La contribution de la Charte des droits fondamentaux de l'Union européenne à la garantie des droits sociaux dans l'ordre juridique communautaire ", précit., p. 42. Cependant, il existe une certaine ambiguïté du propos dès lors que l'auteur estime par la suite que les principes énoncés par la Charte ne sont pas justiciables car progammatoires, idem, p. 47. Contra, Jacqueline DUTHEIL DE LA ROCHÈRE, " La place de la Charte des droits fondamentaux de l'Union dans la Constitution européenne ", précit., p. 238.

352 Pour Laurence BURGORGUE-LARSEN, il existe "une brèche dans l'interprétation de l'invocabilité minimale des "principes" que le juge pourrait habilement saisir afin de ne pas rendre purement formelle ou encore "théorique et illusoire" - pour reprendre un dictum fameux de la Cour de Strasbourg - la consécration du socle social de la Charte ", (" Ombres et Lumières ... ", précit., p. 690).

353 Ibid., p. 690. 
aussi constituer une directive d'interprétation juridictionnelle, l'interdépendance des droits supposant que chaque droit soit interprété dans le contexte de tous les autres : " la CJCE pourrait s'appuyer sur ce principe pour donner plus de poids aux droits sociaux et ainsi justifier un rééquilibrage entre droits sociaux et libertés économiques ${ }^{354}$.

Les contraintes du juge communautaire sont cependant importantes: d'une part, ses interprétations pourraient être neutralisées par les explications qui accompagnent la Charte, et qu'il doit dûment prendre en considération ${ }^{355}$. Les explications ont en effet "la fâcheuse conséquence de congeler la jurisprudence en matière de protection des droits fondamentaux à l'échelle européenne ${ }^{356}$. L'utilisation obligatoire des explications du Praesidium, si elles étaient « la condition sine qua non pour une future valorisation identitaire » ${ }^{357}$ et si elles permettent de minimiser les risques de divergences jurisprudentielles ${ }^{358}$, circonscrivent "considérablement" la marge de manœuvre interprétative des juges ${ }^{359}$. D'autre part, le lien entre les droits fondamentaux et les règles de compétence établi par la Charte (celle-ci est limitée au domaine d'application du droit communautaire), et la méthode systématique qui suppose une interprétation des droits en fonction des règles de compétence établies risquent de limiter la marge de manœuvre de la Cour ${ }^{360}$. Enfin, la Pologne, le RoyaumeUni dès la signature du traité de Lisbonne, et la République tchèque au moment de la ratification, ont obtenu un opt-out formalisé dans le premier cas par un protocole dérogatoire qui porte précisément sur la justiciabilité de la Charte.

La Cour optera-t-elle pour une interprétation autonome, au regard de la logique interne du système qu'elle institue ? ${ }^{361}$ Le caractère récent de l'entrée en vigueur du traité de Lisbonne ne permet pas de répondre pour l'heure à cette interrogation, les prises de position étant trop rares et parcellaires. On notera que la Charte a été invoquée par le juge de l'Union pour renforcer son argumentaire visant à promouvoir certains droits sociaux déjà bien établis dans le droit de l'Union : c'est le cas du principe de non-discrimination en fonction de l'âge, consacré comme un "principe général du droit de l'Union" par une interprétation audacieuse de la clause générale de non-discrimination ${ }^{362}$, et dont la qualité et l'importance ont été confirmées dans un arrêt du 19 janvier 2010, l'arrêt Kücükdeveci. La Cour rappelle sa jurisprudence Mangold et ajoute qu'il « convient de relever que l'article 6, paragraphe 1, TUE énonce que la Charte des droits fondamentaux de l'UE a la même valeur juridique que les traités. Selon l'article 21, paragraphe 1 , de cette charte, "est interdite toute

\footnotetext{
${ }^{354}$ Marie-Claire PONTHOREAU, « Le principe de l'indivisibilité des droits ... », précit., p. 935.

${ }^{355}$ Article 6, § 1, alinéa 3 du TUE.

${ }^{356}$ Laurence BURGORGUE-LARSEN, « Ombres et Lumières ... », précit., pp. 681-682.

357 Ibid.

358 Ibid., p. 672.

359 Ibid., p. 674.

360 Ibid.

361 Ibid.

362 V. supra, 1, sur l'arrêt Mangold, CJCE, 22 novembre 2005, aff. C-144/04, Rec., p. I-19981.
} 
discrimination fondée notamment sur [...] l'âge ${ }^{363}$. De manière sans doute plus audacieuse, et dans un raisonnement qui n'est pas sans rappeler celui tenu par la Cour européenne des droits de l'Homme dans le domaine des droits sociaux ${ }^{364}$, la Cour vient de conclure à la violation d'une disposition de la directive $n^{\circ} 2003 / 88$ concernant certains aspects de l'aménagement du temps de travail ${ }^{365}$ et visant à limiter la durée hebdomadaire du travail. Le requérant contestait une décision de mutation prise par son employeur, dont il estimait qu'elle n'avait été prise qu'en raison du fait qu'il avait demandé une réduction de son temps de travail conformément aux dispositions de la directive. Pour la Cour, une telle mutation forcée vide de toute substance le droit conféré par l'article 6 de la directive relative à la durée maximale de travail hebdomadaire. La Cour estime en outre que "le droit fondamental à une protection juridictionnelle effective, garanti par l'article 47 de la Charte des droits fondamentaux de l'Union européenne, [...], serait substantiellement affecté si un employeur, en réaction à une plainte ou à une action en justice engagée par un travailleur en vue d'assurer le respect des dispositions d'une directive visant à protéger sa sécurité et sa santé, était en droit de prendre une mesure telle que celle en cause au principal. En effet, la crainte de pareille mesure de rétorsion contre laquelle aucun recours juridictionnel ne serait ouvert risquerait de dissuader les travailleurs s'estimant lésés [...] de faire valoir leurs droits par voie juridictionnelle et partant, serait de nature à compromettre gravement la réalisation de l'objectif poursuivi par la directive ${ }^{366}$.

Centrée sur l'Homme, l'Union se fonde aussi désormais sur la citoyenneté, instrument de promotion des droits sociaux dans l'ordre juridique de l'UE.

\section{B. La citoyenneté européenne, nouveau moteur des droits sociaux dans l'ordre juridique de l'UE}

La liberté de circulation et de séjour, droit cardinal de la citoyenneté européenne, associée à une conception extensive du principe d'égalité, permet l'émergence d'une "citoyenneté sociale" (1), dont les limites sont sans cesse réduites ou redéfinies par la juridiction de l’Union (2).

\section{1) L'émergence d'une " citoyenneté sociale "}

La doctrine, au départ réticente à reconnaître une réelle valeur ajoutée à la citoyenneté européenne, s'intéresse depuis peu de manière approfondie à la dimension sociale de la notion ${ }^{367}$. Depuis une dizaine d'années, les arrêts de la CJCE sont très souvent relatifs à l'octroi de droits sociaux aux ressortissants

\footnotetext{
363 CJUE, 19 janvier 2010, Seda Kücükdeveci, aff. C-555/07, point 22.

364 V. Diane ROMAN, infra.

365 Directive 2003/88/CE du 4 novembre 2003, JO L 299, p. 9.

366 CJCE, 14 octobre 2010, Günter Fuß, aff. C-243/09, point 66.

367 Les deux derniers exemples les plus significatifs sont les thèses précitées de Sandrine MAILLARD et de Claire MARZO.
} 
communautaires amenés à faire usage de leur liberté de circulation, la Cour n'ayant de cesse de combler les lacunes du droit dérivé ${ }^{368}$. La doctrine a mis l'accent sur l'extension radicale de l'applicabilité du principe d'égalité, dans des décisions devenues célèbres, comme l'arrêt Martinez Sala ${ }^{369}$, qui appuie la reconnaissance du droit à un avantage social (une allocation d'éducation pour enfant) sur le statut de citoyenne de la requérante, ressortissante espagnole, inactive, résidant en Allemagne, ou plus récemment, l'important arrêt Rudy Grzelczyk en date du 20 septembre $2001^{370}$, rendu au sujet du refus de la Belgique d'accorder le minimum de moyens d'existence ("minimex") à un ressortissant communautaire de nationalité française, poursuivant ses études universitaires en Belgique, et réclamant le bénéfice de cette aide sociale pour financer sa quatrième et dernière année d'étude. Dans l'arrêt Marie-Nathalie $D^{\prime}$ Hoop $^{371}$, la Cour juge inapplicable le droit communautaire relatif aux travailleurs, qui exige que l'individu ait déjà accédé au marché du travail pour pouvoir prétendre à une allocation de chômage. C'est donc au nom du principe d'égalité et de la citoyenneté européenne que la requérante peut obtenir une allocation l'aidant dans sa recherche d'un premier emploi, qui plus est dans son propre Etat ${ }^{372}$. Dans l'arrêt Collins du 23 mars 2004, la Cour inclut dans le champ d'application du traité une prestation de nature financière destinée à faciliter l'accès à l'emploi sur le marché du travail d'un Etat membre ${ }^{373}$. Finalement, cette jurisprudence, qui se développe alors même que les Etats gardent leurs compétences en matière de sécurité sociale ou d'éducation, aboutit à une extension remarquable de l'accès aux prestations sociales relevant de la solidarité nationale aux citoyens inactifs, étudiants ou sans emploi. L’idée sousjacente est que «bien que les gouvernements nationaux gardent la compétence exclusive pour régir des matières telles que la sécurité sociale ou la politique d'éducation, ils ne peuvent restreindre l'exercice des droits garantis par le traité

\footnotetext{
368 Norbert REICH et Selvita HARBACEVICA, "Citizenship and Family on Trial : a Fairly Optimistic Overview of Recent Court Practice With Regard to Free Movement of Persons ", C.M.L.Rev., juin 2003, vol. 40, n 3, p. 627.

369 CJCE, 12 mai 1998, Martinez Sala, aff. C-85/96, Rec., p. I-2691. V. en ce sens Siofra O'LEARY, "Putting Flesh on the Bones of European Union Citizenship », E.L.Rev., 1999, pp. 77-78, l'auteur estimant que l'arrêt "confirms that Union citizenship explodes the "linkages" which E.C. law previously required for the principle of non discrimination to apply, namely performance or involvement in an economic activity as workers, established persons or providers and recipients of services, preparation for a future economic activity as a student or stagiaire or some sort of relationship with an economic actor as a family member or dependant ".

370 CJCE, 20 septembre 2001, Rudy Grzelczyk c. Centre public d'aide sociale d'Ottignies-Louvainla-Neuve, aff. C-184/99, Rec., p. I-6193.

371 CJCE, 11 juillet 2002, Marie-Nathalie D’Hoop c. Office national de l'emploi, aff. C-224/98, Rec., p. 6191. Sur cet arrêt, V. le commentaire d'Anastasia ILIOPOULOU et Helen TONER, "A New Approach to Discrimination against Free Movers? D'Hoop v/ Office National de l'Emploi ", E.L.Rev., 2003, vol. 28, n 3, pp. 389-397.

372 Sur l'importance de ces jurisprudences et la notion de discrimination à rebours, V. Myriam BENLOL CARABOT, Les fondements juridiques de la citoyenneté européenne, Bruylant, 2006, pp. 517-538. Sur le principe de non-discrimination, Anastasia ILIOPOULOU, Libre circulation et non-discrimination, éléments du statut de citoyen de l’Union européenne, Bruylant, 2008.

373 CJCE, 23 mars 2004, Collins, aff. C-138/02, Rec., p. I-2703.
} 
afin d'assurer que les fonds et les ressources financières pertinents ne bénéficient qu'à leurs propres ressortissants nationaux $»^{374}$.

La jurisprudence de la CJCE consacre sans doute l'émergence d'une «citoyenneté sociale». L'autonomisation des droits attachés à la citoyenneté européenne et la déconnexion toujours plus grande de la faculté d'exercer les libertés communautaires de l'exercice d'une activité économique permettent de donner tout son sens à cette notion ${ }^{375}$. La Cour recherche, par l'intermédiaire de la citoyenneté européenne, des discriminations indirectes qui lui permettent de mettre en œuvre les conditions d'une véritable intégration sociale ${ }^{376}$.

\section{2) Des limites clairement circonscrites}

Le droit dérivé autorise les Etats membres à déroger au droit de séjour du citoyen européen, ainsi qu'à l'octroi de prestations sociales. La Cour juge par exemple légitime pour un Etat de vouloir s'assurer de l'existence d'un lien réel entre le demandeur d'allocations et le marché géographique du travail en cause, tout comme elle ne peut que prendre acte des limitations au droit de séjour, le citoyen de l'Union ne pouvant demeurer sur le territoire d'un Etat membre pour plus de trois mois que s'il dispose pour lui et sa famille de ressources suffisantes pour ne pas devenir une charge pour le système d'assistance sociale de l'Etat membre d'accueil ${ }^{377}$. Ces limites font cependant l'objet d'une définition communautaire, la Cour ayant progressivement précisé, s'agissant par exemple d'une aide couvrant les frais d'entretien des étudiants, qu'il était légitime pour un Etat membre de n'octroyer une telle aide qu'aux étudiants ayant démontré un certain degré d'intégration dans la société de cet Etat»378. Le critère de résidence peut ainsi conditionner l'accès à une allocation de recherche d'emploi ${ }^{379}$, à une allocation de chômage ${ }^{380}$, à un prêt étudiant ${ }^{381}$, à une bourse d'entretien aux étudiants ${ }^{382}$. L'utilisation toujours plus importante du critère de

374 Conclusions de l'Avocat général POIARES MADURO présentées le 10 octobre 2007, affaire Jundt, aff. C-281/06, point 19.

375 Très nombreuses références doctrinales: entre autres, V. Claire MARZO, "Vers une citoyenneté sociale européenne?", Droit social, février 2007, pp. 218-224; Nelly ACH, "La citoyenneté européenne au service d'une Europe sociale ", Journal des tribunaux Droit européen, 2006, fasc. 129, pp. 129-134 ; Pierre RODIERE, « Droit social, libre circulation des personnes et citoyenneté européenne dans la jurisprudence de la Cour de justice », RTDE, 2006, pp. 142-163; Florence ZAMPINI, "La jurisprudence de la Cour de justice et les étudiants : du droit à la nondiscrimination en matière d'accès à l'enseignement universitaire dans l'Etat d'accueil à l'octroi de prestations sociales ", RAE, 2005, pp. 63-82.

376 Sur ce point et pour l'analyse d'une jurisprudence fournie, V. Claire MARZO, La dimension sociale de la citoyenneté européenne, Thèse IUE Florence, 2009, dactyl., p. 324 et s.

377 Article 7, 1, b), de la directive $\mathrm{n}^{\circ} 2004 / 38$ du 29 avril 2004 relative au droit des citoyens de l'Union et des membres de leurs familles de circuler et de séjourner librement sur le territoire des Etats membres, JOUE L 229 du 29 juin 2004, p. 35.

378 CJCE, 15 mars 2005, Bidar, aff. C-209/03, Rec., p. I-2119, point 57.

379 CJCE, Collins, précit.

380 CJCE, 18 juillet 2006, De Cuyper, aff. C-406/04, Rec., p. I-6947.

381 CJCE, Bidar, précit.

382 CJCE, 18 novembre 2008, Förster, aff. C-158/07, Rec., p. I-8507. 
résidence permet ainsi l'émergence progressive d'un territoire communautaire ${ }^{383}$ et la concrétisation d'une solidarité sociale communautaire.

Cette solidarité sociale trouve plus particulièrement à s'exprimer dans l'interprétation que fait la Cour de la condition de "ressources suffisantes" posée par le droit dérivé au maintien du droit de séjour dans l'Etat membre d'accueil. La Cour propose une interprétation constructive de cette condition dans l'arrêt Trojani, estimant que « si les États membres peuvent conditionner le séjour d'un citoyen de l'Union économiquement non actif à la disponibilité de ressources suffisantes, il n'en découle pas pour autant qu'une telle personne ne peut pas bénéficier, pendant son séjour légal dans l'État membre d'accueil, du principe fondamental relatif à l'égalité de traitement tel que consacré à l'article $12 \mathrm{CE}$ ", ce qui rend donc contraire au droit communautaire toute mesure d'éloignement ${ }^{384}$. Dans un arrêt récent, la Cour a été plus loin encore, en jugeant qu'une personne travaillant par intermittence et n'ayant pas de ressources propres suffisantes pouvait néanmoins séjourner sur le territoire d'un Etat membre afin de permettre à son enfant, dont elle a la garde, de poursuivre ses études dans cet Etat ${ }^{385}$.

Tardive et encore lacunaire, la prise en compte des droits sociaux dans l'ordre juridique de l'UE n'en est pas moins indéniable. Restent bien sûr les tensions propres au processus d'intégration communautaire, les finalités économiques des traités rendant laborieuse toute conceptualisation des droits sociaux sur un autre fondement. Le processus de construction de la citoyenneté européenne, visant à rendre plus humaine l'Union tout en en réservant le privilège aux nationaux des Etats membres, illustre avec acuité ces ambigüités.

\section{Pour citer cet article}

Myriam Benlolo-Carabot «Les droits sociaux dans l'ordre juridique de l'Union Européenne. Entre instrumentalisation et 'fondamentalisation'?», La Revue des Droits de l'Homme, juin 2012

http://revdh.files.wordpress.com/2012/06/les-droits-sociaux-dans-lordre-juridique-de-lunioneuropc3a9enne.pdf

\footnotetext{
383 Myriam BENLOLO CARABOT, précit., p. 582 et s.

${ }^{384}$ CJCE, 7 septembre 2004, Trojani, aff. C-456/02, Rec. 2004, p. I-7573, point 40.

385 CJUE, 23 février 2010, Maria Teixeira, aff. C-480/08; dans le même sens, V. l'arrêt du même jour, Ibrahim, C-310/08.
} 